Bayesian Analysis (2019)

14, Number 1, pp. 1-28

\title{
Bayesian Method for Causal Inference in Spatially-Correlated Multivariate Time Series
}

\author{
Bo Ning*, Subhashis Ghosal ${ }^{\dagger}$, and Jewell Thomas ${ }^{\ddagger}, \S$
}

\begin{abstract}
Measuring the causal impact of an advertising campaign on sales is an essential task for advertising companies. Challenges arise when companies run advertising campaigns in multiple stores which are spatially correlated, and when the sales data have a low signal-to-noise ratio which makes the advertising effects hard to detect. This paper proposes a solution to address both of these challenges. A novel Bayesian method is proposed to detect weaker impacts and a multivariate structural time series model is used to capture the spatial correlation between stores through placing a $\mathcal{G}$-Wishart prior on the precision matrix. The new method is to compare two posterior distributions of a latent variable - one obtained by using the observed data from the test stores and the other one obtained by using the data from their counterfactual potential outcomes. The counterfactual potential outcomes are estimated from the data of synthetic controls, each of which is a linear combination of sales figures at many control stores over the causal period. Control stores are selected using a revised Expectation-Maximization variable selection (EMVS) method. A two-stage algorithm is proposed to estimate the parameters of the model. To prevent the prediction intervals from being explosive, a stationarity constraint is imposed on the local linear trend of the model through a recently proposed prior. The benefit of using this prior is discussed in this paper. A detailed simulation study shows the effectiveness of using our proposed method to detect weaker causal impact. The new method is applied to measure the causal effect of an advertising campaign for a consumer product sold at stores of a large national retail chain.
\end{abstract}

MSC 2010 subject classifications: $62 \mathrm{~F} 15$.

Keywords: advertising campaign, Bayesian variable selection, causal inference, graphical model, stationarity, time series.

\section{Introduction}

Advertising is thought to impact sales in markets. MaxPoint Interactive Inc. (MaxPoint), an online advertising company, ${ }^{1}$ is interested in measuring the sales increases

\footnotetext{
*Department of Statistics, North Carolina State University, 2501 Founders Drive, Raleigh, North Carolina 27695, USA, bning@ncsu.edu

${ }^{\dagger}$ Department of Statistics, North Carolina State University, 2501 Founders Drive, Raleigh, North Carolina 27695, USA, sghosal@ncsu.edu

${ }^{\ddagger}$ Department of English \& Comparative Literature, The University of North Carolina at Chapel Hill, CB \#3520 Greenlaw Hall, Chapel Hill, North Carolina 27599, USA, jewellt@live.unc.edu

$\S$ Jewell Thomas is a former Staff Data Scientist at MaxPoint Interactive Inc., 3020 Carrington Mill Blvd, Morrisville, North Carolina 27560, USA

${ }^{1}$ The methodology developed and presented in this paper is not connected to any commercial products currently sold by MaxPoint.
} 
associated with running advertising campaigns for products distributed through brickand-mortar retail stores.

The dataset provided by Maxpoint was obtained as follows: MaxPoint ran an advertising campaign at 627 test stores across the United States. An additional 318 stores were chosen as control stores. Control stores were not targeted in the advertising campaign. The company collected weekly sales data from all of these stores for 36 weeks before the campaign began and for the 10 weeks in which the campaign was conducted. The time during which the campaign was conducted is known. The test stores and the control stores were randomly selected from different economic regions across the U.S.. Figure 1 shows an example of the locations of stores in the State of Texas. ${ }^{2}$

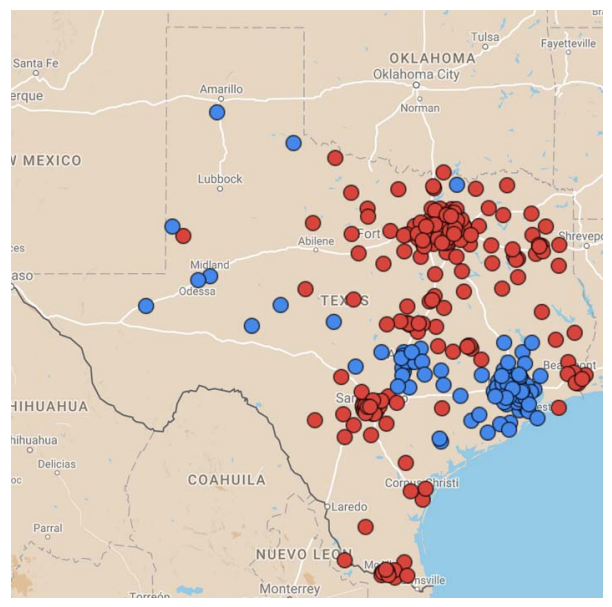

Figure 1: An example of test and control store locations in the State of Texas (Google Maps, 2017). The red dots represent the locations of the test stores; the blue dots represent the locations of the control stores.

To the best of our knowledge, the work of Brodersen et al. (2015) is the most related one to the present study. Their method can be described as follows. For each test store, they first split its time series data into two parts: before and during a causal impact (in our case, the impact is the advertising campaign). Then, they used the data collected before the impact to predict the values during the causal period. At the same time, they applied a stochastic search variable selection (SSVS) method to construct a synthetic control for that store. The counterfactual potential outcomes (Rubin, 2005) are the sum of the predicted values and the data from the synthetic control. Clearly, the potential outcomes of the store exposed to advertising were the observed data. Finally, they compared the difference between the two potential outcomes and took the average of differences across different time points. The averaged difference is a commonly used causal estimand that measures the temporal average treatment effect (Bojinov and Shephard, 2017).

\footnotetext{
${ }^{2}$ Note: The locations of the stores shown in the figure are not associated with any real datasets collected by MaxPoint.
} 
The method proposed by Brodersen et al. (2015) is novel and attractive; however, it cannot directly apply to analyze our dataset due to the following three reasons: (1) Many causal impacts in our dataset are weak. The causal estimand that Brodersen et al. (2015) used often fails to detect them; (2) The test stores within an economic region are spatially correlated as they share similar demographic information. Using Brodersen et al. (2015)'s method would not allow to consider the spatial correlation between stores; (3) The SSVS method is computationally slow because it requires sampling from a large model space consisting of $2^{p}$ possible combinations of $p$ control stores. In the following, we will discuss our proposed method for addressing these three difficulties.

First, we propose a new method for detecting weaker causal impacts. The method compares two posterior distributions of the latent variables of the model, where one distribution is computed by conditioning the observed data and the other one is computed by conditioning the counterfactual potential outcomes. We use the one-sided Kolmogorov-Smirnov (KS) distance to quantify the distance between the two posterior distributions.

The new method can successfully detect weaker impacts because it compares two potential outcomes at the latent variable level; while the commonly used method compares them at the observation level. Since the observed data often contain "inconvenient" components - such as seasonality and random errors - which inflate the uncertainty of the estimated causal effect, the commonly used method may fail to detect weaker impacts. In the simulation study, we show that the new method outperforms the commonly used method even when the model is slightly misspecified.

The causal estimand in the new method is different from the causal estimand of the commonly used method. The former one measures the temporal average treatment effect using the KS distance between two posterior distributions and the latter measures that effect using the difference between two potential outcomes. Formal definitions of the two causal estimands are provided in Section 2.

Secondly, we use a multivariate version of a structural time series model (Harvey and Peters, 1990) to model the sales data of test stores by allowing pooling of information among those stores that locate in geographically contiguous economic regions. This model enjoys a few advantages that make it especially suitable for our causal inference framework. First, the model is flexible to adapt to different structures of the latent process. Secondly, it can be written as a linear Gaussian state-space model and exact posterior sampling methods can be carried out by applying the Kalman filter and simulation smoother algorithm proposed by Durbin and Koopman (2002, 2012). Thirdly, it is relatively easy to deal with missing data due to the use of the Kalman filter and backward smoothing (KFBS) algorithm. The imputing process can be naturally incorporated into the Markov chain Monte Carlo (MCMC) loop.

Since test stores are correlated, the number of parameters in the covariance matrix grows quadratically with the dimension. Consequently, there will not be enough data to estimate all these parameters. In our approach, we reduce the number of parameters by imposing sparsity based on a given spatial structure (Smith and Fahrmeir, 2007; Barber and Drton, 2015; Li et al., 2015). We consider a graphical model structure for dependence based on geographical distances between stores. If the distance between two stores is very 
large, we treat them conditionally independent given other stores. In terms of a graphical model, this is equivalent to not put an edge between them. We denote the corresponding graph by $\mathcal{G}$. Note that $\mathcal{G}$ is given in our setting and is completely determined by the chosen thresholding procedure. We use a graphical Wishart prior with respect to the given graph $\mathcal{G}$, in short a $\mathcal{G}$-Wishart prior (Roverato, 2002), to impose sparsity on the precision matrix. One advantage is that this prior is conjugate for a multivariate normal distribution. If $\mathcal{G}$ is decomposable, sampling from a conjugate $\mathcal{G}$-Wishart posterior is relatively easy due to an available closed form expression for the normalizing constant in the density (Lauritzen, 1996; Roverato, 2000, 2002). However, if $\mathcal{G}$ is non-decomposable, the normalizing constant does not usually have a simple closed form (see however; Uhler et al., 2017), and thus one cannot easily sample directly from its posterior. In such a situation, an approximation for the normalizing constant is commonly used (Atay-Kayis and Massam, 2005; Mitsakakis et al., 2011; Wang and Li, 2012; Khare et al., 2015). A recent method introduced by Mohammadi and Wit (2015) is a birth-death Markov chain Monte Carlo (BDMCMC) sampling method. It uses a trans-dimension MCMC algorithm that transforms sampling of a high-dimensional matrix to lower dimensional matrices, thus improving efficiency when working with large precision matrices.

In a multivariate state-space model, the time dynamics are described by a multivariate stochastic trend, usually an order-one vector autoregressive (VAR(1)) process (de Jong, 1991; de Jong and Chu-Chun-Lin, 1994; Koopman, 1997; Durbin and Koopman, 2002). To use a order $p$ vector autoregression with order $q$ moving average $(\operatorname{VARMA}(p, q))$ process, with $p>1, q \geq 0$, is also possible and the choice of $p, q$ can be made based on data (e.g., chosen by the Bayesian Information Criterion). However, the larger the $p$ and $q$ are, the larger the number of parameters that need to be estimated. For the sake of tractability, we treat the hidden process as a VAR(1) process throughout the paper.

Putting stationarity constraints on the VAR(1) process is necessary to prevent the prediction intervals from becoming too wide to be useful. However, constructing an appropriate prior complying with the constraints is not straightforward. Gelfand et al. (1992) proposed a naive approach that puts a conjugate prior on the vector autoregressive parameter to generate samples and only keep the samples meeting the constraints. However, it can be highly inefficient when many draws from the posterior correspond to nonstationary processes. A simple remedy is to project these nonstationary draws on the stationarity region to force them to meet the constraints (Gunn and Dunson, 2005). However, the projection method is somewhat unappealing from a Bayesian point of view because it would make the majority of the projected draws have eigenvalues lying on the boundary of the corresponding space (Galindo-Garre and Vermunt, 2006; Roy et al., 2016). We instead follow the recently proposed method of Roy et al. (2016) to decompose the matrix into several unrestricted parameters so that commonly used priors can be put on those parameters. While conjugacy will no longer be possible, efficient algorithms for drawing samples from the posterior distribution are available.

Thirdly, to accelerate the computational speed of selection control stores, we suggest using a revised version of the Expectation-Maximization variable selection (EMVS) method (Ročková and George, 2014). The model uses an Expectation-Maximization (EM) algorithm that is faster and does not need to search $2^{p}$ possible combinations. 
It is worth mentioning that there are many other popular methods for constructing a synthetic control, such as the synthetic control method proposed by Abadie and Gardeazabal (2003), the difference-in-differences method (Abadie, 2005; Bonhomme and Sauder, 2011; Donald and Lang, 2007), and the matching method (Stuart, 2010). Moreover, Doudchenko and Imbens (2016) provided a nice discussion on the advantages and disadvantages of each method. Unlike these methods, there are two advantages of using our proposed method: It does not need to have a prior knowledge about the relevant control stores, the process of selecting control stores is completely driven by data and can be easily incorporated into a Bayesian framework. It provides a natural model-based causal inference by viewing counterfactual potential outcomes as missing values and generating predicting credible intervals from their posterior predictive distributions, and finally providing a quantitative measure for the strength of the causal effect (Rubin, 2005).

We apply our method on both simulated datasets and the real dataset provided by MaxPoint. In the simulation study, we compare the new method with the method proposed by Brodersen et al. (2015).

The rest of the paper is organized as follows. Section 2 introduces causal assumptions and causal estimands. Section 3 describes the model and the priors. Section 4 describes posterior computation techniques. Section 5 introduces our proposed new approach to infer causal effects in times series models. Simulation studies are conducted in Section 6 . In Section 7, the proposed method is applied on a real dataset from an advertising campaign conducted by MaxPoint. Finally, Section 8 concludes with a discussion.

\section{Causal assumptions and causal estimands}

This section includes three parts. First, we will introduce the potential outcomes framework. Secondly, we shall discuss three causal assumptions. Finally, we shall define two causal estimands, one of them is new.

The potential outcomes framework is widely used in causal inference literature (Rubin, 1974, 2005; Ding and Li, 2017). Potential outcomes are defined as the values of an outcome variable at a future point in time after treatment under two different treatment levels. Clearly, at most one of the potential outcomes for each unit can be observed, and the rest are missing (Holland, 1986; Rubin, 1977; Imbens and Rubin, 2015). The missing values can be predicted using statistical methods. In the paper, we predict the values using the data from a synthetic control that is constructed from several control stores.

Based on the potential outcomes framework, we conduct the causal inference. There are three assumptions need to make for conducting the inference. They are,

1. The stable unit treatment value assumption (SUTVA);

2. The strong ignorability assumption on the assignment mechanism;

3. The trend stays stable in the absence of treatment for each test store. 
The SUTVA contains two sub-assumptions: no interference between units and no different versions of a treatment (Rubin, 1974). The first assumption is reasonable because the stores did not interact with each other after the advertising was assigned. As Rosenbaum (2007) pointed out, "interference is distinct from statistical dependence produced by pretreatment clustering." Since the spatial correlation between test stores is produced by pretreatment clustering, it is different from the interference between stores. The second assumption is also sensible because we assume that there are no multiple versions of the advertising campaign. For example, the advertising campaign is not launched across multiple channels.

The strong ignorability assumption also contains two parts: unconfoundedness and positivity (Ding and Li, 2017). Unconfoundedness means that the treatment is assigned randomly and positivity means that the probability for each store being assigned is positive. In our study, we assume the company randomly assigned advertising to stores and each store has an equal probability of being assigned.

The last assumption says that the counterfactual potential outcomes in the absence of the advertising in test stores are predictable.

Now, we shall introduce some notations before defining causal estimands. Let $n$ be the total number of test stores to which the advertising were assigned. The $i$-th test store has $p_{i}$ control stores (stores did not assigned with the advertising), $i=1, \ldots, n$. The total number of control stores are denoted as $p, p=\sum_{i=1}^{n} p_{i}$. The length of the time series data is $T+P$. Let $1, \ldots, T$ be the periods before running the advertising campaign and $T+1, \ldots, T+P$ be the periods during the campaign. Let $\boldsymbol{w}_{t}=\left(w_{1 t}, \ldots, w_{n+p, t}\right)^{\prime}$ be a vector of treatment at time $t=T+1, \ldots, T+P$, with each $w_{i t}$ being a binary variable. The treatment assignment is time-invariant, so $\boldsymbol{w}_{t}=\boldsymbol{w}$. For stores assigned with advertising, we denote the sales value for the $i$-th store at times $t$ as $y_{i t}$. Let $y_{i t}^{\text {obs }}$ be the observed data and $y_{i t}^{\text {cf }}$ be the counterfactual potential outcomes which are missing. We let $\boldsymbol{Y}_{t}^{\mathrm{obs}}=\left(y_{1 t}^{\mathrm{obs}}, \ldots, y_{n t}^{\mathrm{obs}}\right)^{\prime}$ and $\boldsymbol{Y}_{t}^{\mathrm{cf}}=\left(y_{1 t}^{\mathrm{cf}}, \ldots, y_{n t}^{\mathrm{cf}}\right)^{\prime}$ respectively be the observed and missing potential outcomes for $n$ test stores at time $t, t=1, \ldots, T+P$. Clearly, $\boldsymbol{Y}_{t}^{\text {obs }}=\boldsymbol{Y}_{t}^{\text {cf }}$ when $t=1, \ldots, T$. We define $\boldsymbol{Y}_{T+1: T+P}^{\text {obs }}=\left(\boldsymbol{Y}_{T+1}^{\mathrm{obs}}, \ldots, \boldsymbol{Y}_{T+P}^{\mathrm{obs}}\right)^{\prime}$ and $\boldsymbol{Y}_{T+1: T+P}^{\mathrm{cf}}=\left(\boldsymbol{Y}_{T+1}^{\mathrm{cf}}, \ldots, \boldsymbol{Y}_{T+P}^{\mathrm{cf}}\right)^{\prime}$.

We first define the causal estimand of a commonly used method. For the $i$-th test store, the commonly used causal estimand is defined as

$$
\frac{1}{P} \sum_{t=T+1}^{T+P}\left(y_{i t}^{\mathrm{obs}}-y_{i t}^{\mathrm{cf}}\right),
$$

which is the temporal average treatment effects (Bojinov and Shephard, 2017) at $P$ time points. In our setting, the treatment effects for $n$ test stores are defined as

$$
\frac{1}{P} \sum_{t=T+1}^{T+P}\left(\boldsymbol{Y}_{t}^{\mathrm{obs}}-\boldsymbol{Y}_{t}^{\mathrm{cf}}\right) .
$$

To introduce our new causal estimand, let $x_{i t}$ be the data for the synthetic control for the $i$-th test store at time $t$. Recall that the data of a synthetic control is a weighted 
sum of the sales from several control stores. Define $\boldsymbol{X}_{1: T+P}=\left(\boldsymbol{X}_{1}, \ldots, \boldsymbol{X}_{T+P}\right)$, where $\boldsymbol{X}_{t}$ is an $n \times p$ matrix containing data from $p$ control stores at time $t$. Let $\mu_{i t}$ be a latent variable of a model, which is of interest. Define $\boldsymbol{\mu}_{t}=\left(\boldsymbol{\mu}_{1 t}, \ldots, \boldsymbol{\mu}_{n t}\right)$ which is an $n \times 1$ vector. We let

$$
p\left(\sum_{t=T+1}^{T+P} \boldsymbol{\mu}_{t} \mid \boldsymbol{Y}_{1: T+P}^{\mathrm{obs}}, \boldsymbol{X}_{1: T+P}\right),
$$

be the posterior distribution of the latent variable conditional on $\boldsymbol{Y}_{1: T+P}^{\text {obs }}$ and $\boldsymbol{X}_{1: T+P}$, and

$$
p\left(\sum_{t=T+1}^{T+P} \boldsymbol{\mu}_{t} \mid \boldsymbol{Y}_{1: T}^{\mathrm{obs}}, \boldsymbol{Y}_{T+1: T+P}^{\mathrm{cf}}, \boldsymbol{X}_{1: T+P}\right),
$$

be the distribution conditional on $\boldsymbol{Y}_{1: T+P}^{\mathrm{cf}}$ and $\boldsymbol{X}_{1: T+P}$.

The new causal estimand is defined as the one-sided Kolmogorov-Smirnov (KS) distance between the two distributions for $i$-th store, which can be expressed as

$$
\begin{aligned}
\sup _{x} & {\left[\mathcal{F}\left(\sum_{t=T+1}^{T+P} \mu_{i t} \leq x \mid y_{i, 1: T}^{\mathrm{obs}}, y_{i, T+1: T+P}^{\mathrm{cf}}, x_{i, 1: T+P}\right)\right.} \\
& \left.-\mathcal{F}\left(\sum_{t=T+1}^{T+P} \mu_{i t} \leq x \mid y_{i, 1: T+P}^{\mathrm{obs}}, x_{i, 1: T+P}\right)\right],
\end{aligned}
$$

where $\mathcal{F}(\cdot)$ stands for the corresponding cumulative distribution function. In our setting, since test stores are spatially correlated, the causal effect of the $i$-th test store is defined as

$$
\begin{aligned}
\sup _{x} & {\left[\mathcal{F}\left(\sum_{t=T+1}^{T+P} \mu_{i t} \leq x \mid \boldsymbol{Y}_{1: T}^{\mathrm{obs}}, \boldsymbol{Y}_{T+1: T+P}^{\mathrm{cf}}, \boldsymbol{X}_{1: T+P}\right)\right.} \\
& \left.-\mathcal{F}\left(\sum_{t=T+1}^{T+P} \mu_{i t} \leq x \mid \boldsymbol{Y}_{1: T+P}^{\mathrm{obs}}, \boldsymbol{X}_{1: T+P}\right)\right] .
\end{aligned}
$$

A larger value of the one-sided KS distance implies a potentially larger scale of causal impact. An impact is declared to be significant if the one-sided KS distance is larger than its corresponding threshold. The threshold is calculated based on several datasets that are randomly drawn from the posterior predictive distribution of (3) (See Section 5 for more details.)

We would like to mention that although the proposed method is applied to a multivariate time series model in this paper, even in the context of a univariate model, the idea of comparing posterior distributions of latent variables appears to be new. Generally speaking, this idea can be adopted into many other applications with different Bayesian models as long as these models are described in terms of latent variables. 


\section{Model and prior}

\subsection{Model}

We consider a multivariate structural time series model given by (to simplify the notation, we use $\boldsymbol{Y}_{t}$ instead of $\boldsymbol{Y}_{t}^{\text {obs }}$ in the current and the following sections),

$$
\boldsymbol{Y}_{t}=\boldsymbol{\mu}_{t}+\boldsymbol{\delta}_{t}+\boldsymbol{X}_{t} \boldsymbol{\beta}+\boldsymbol{\epsilon}_{t}
$$

where $\boldsymbol{Y}_{t}, \boldsymbol{\mu}_{t}, \boldsymbol{\delta}_{t}$ and $\boldsymbol{\epsilon}_{t}$ are $n \times 1$ vectors standing for the response variable, trend, seasonality and measurement error respectively. $n$ is the number of test stores, $\boldsymbol{X}_{t}$ is an $n \times p$ matrix containing data from $p$ control stores at time $t$ and $\boldsymbol{\beta}$ is a sparse $p \times 1$ vector of regression coefficients, where $p$ can be very large. We allow each response in $\boldsymbol{Y}_{t}$ to have different number of control stores, and write

$$
\boldsymbol{X}_{t}=\left(\begin{array}{cccccccccc}
x_{11, t} & \cdots & x_{1 p_{1}, t} & 0 & \cdots & 0 & \cdots & 0 & \cdots & 0 \\
0 & \cdots & 0 & x_{21, t} & \cdots & x_{2 p_{2}, t} & \cdots & 0 & \cdots & 0 \\
& \ddots & & & \ddots & & & & \ddots & \\
0 & \cdots & 0 & 0 & \cdots & 0 & \cdots & x_{n 1, t} & \cdots & x_{n p_{n}, t}
\end{array}\right),
$$

with $\sum_{i=1}^{n} p_{i}=p$. Let $\gamma=\left(\gamma_{1}, \ldots, \gamma_{p}\right)$ be the indicator variable such that $\gamma_{j}=1$ if and only if $\beta_{j} \neq 0 . \boldsymbol{\epsilon}_{t}$ is an independent and identically distributed (i.i.d) error process.

The trend of the time series is modeled as

$$
\boldsymbol{\mu}_{t+1}=\boldsymbol{\mu}_{t}+\boldsymbol{\tau}_{t}+\boldsymbol{u}_{t},
$$

where $\boldsymbol{\tau}_{t}$ is viewed as a term replacing the slope of the linear trend at time $t$ to allow for a general trend, and $\boldsymbol{u}_{t}$ is an i.i.d. error process. The process $\boldsymbol{\tau}_{t}$ can be modeled as a stationary $\operatorname{VAR}(1)$ process, driven by the equation

$$
\boldsymbol{\tau}_{t+1}=\boldsymbol{D}+\boldsymbol{\Phi}\left(\boldsymbol{\tau}_{t}-\boldsymbol{D}\right)+\boldsymbol{v}_{t},
$$

where $\boldsymbol{D}$ is an $n \times 1$ vector and $\boldsymbol{\Phi}$ is an $n \times n$ matrix of the coefficients of the $\operatorname{VAR}(1)$ process with eigenvalues having modulus less than 1 . If no stationarity restriction is imposed on $\boldsymbol{\tau}_{t}$, we model it by

$$
\boldsymbol{\tau}_{t+1}=\boldsymbol{\tau}_{t}+\boldsymbol{v}_{t}
$$

where $\boldsymbol{v}_{t}$ is an i.i.d. error process.

The seasonal component $\boldsymbol{\delta}_{t}$ in (5) is assumed to follow the evolution equation

$$
\boldsymbol{\delta}_{t+1}=-\sum_{j=0}^{S-2} \boldsymbol{\delta}_{t-j}+\boldsymbol{w}_{t},
$$

where $S$ is the total length of a cycle and $\boldsymbol{w}_{t}$ is an i.i.d. error process. For example, for an annual dataset, $S=12$ represents the monthly effect while $S=4$ represents the quarterly effect. This equation ensures that the summation of $S$ time periods of each variable has expectation zero. 
We assume that the residuals of (5)-(9) are mutually independent and time invariant, and are distributed as multivariate normals with mean $\mathbf{0}_{n \times 1}$ and covariance matrices $\boldsymbol{\Sigma}, \boldsymbol{\Sigma}_{u}, \boldsymbol{\Sigma}_{v}$ and $\boldsymbol{\Sigma}_{w}$ respectively.

By denoting parameters $\boldsymbol{\alpha}_{t}=\left(\boldsymbol{\mu}_{t}^{\prime}, \boldsymbol{\tau}_{t}^{\prime}, \boldsymbol{\delta}_{t}^{\prime}, \cdots, \boldsymbol{\delta}_{t-S+2}^{\prime}\right)^{\prime}$ and $\boldsymbol{\eta}_{t}=\left(\boldsymbol{u}_{t}^{\prime}, \boldsymbol{v}_{t}^{\prime}, \boldsymbol{w}_{t}^{\prime}\right)^{\prime}$, the model can be represented as a linear Gaussian state-space model

$$
\begin{aligned}
\boldsymbol{Y}_{t} & =\boldsymbol{z} \boldsymbol{\alpha}_{t}+\boldsymbol{X}_{t} \boldsymbol{\beta}+\boldsymbol{\epsilon}_{t}, \\
\boldsymbol{\alpha}_{t+1} & =\boldsymbol{c}+\boldsymbol{T} \boldsymbol{\alpha}_{t}+\boldsymbol{R} \boldsymbol{\eta}_{t},
\end{aligned}
$$

where $\boldsymbol{z}, \boldsymbol{c}, \boldsymbol{T}$ and $\boldsymbol{R}$ can be rearranged accordingly based on the model (5)-(9); and $\boldsymbol{\epsilon}_{t} \sim \mathcal{N}(\mathbf{0}, \boldsymbol{\Sigma}), \boldsymbol{\eta} \sim \mathcal{N}(\mathbf{0}, \boldsymbol{Q}), \boldsymbol{Q}=\operatorname{bdiag}\left(\boldsymbol{\Sigma}_{u}, \boldsymbol{\Sigma}_{v}, \boldsymbol{\Sigma}_{w}\right)$ are mutually independent; here and below "bdiag" refers to a block-diagonal matrix with entries as specified. If $\boldsymbol{\tau}_{t}$ is a nonstationary process in (7), then we set $\boldsymbol{c}=\mathbf{0}$.

\subsection{Prior}

We will now discuss the priors for the parameters in the model. We separate the parameters into four blocks: the time varying parameter $\boldsymbol{\alpha}_{t}$, the stationarity constraint parameters $\boldsymbol{D}$ and $\boldsymbol{\Phi}$, the covariance matrices of the error terms $\boldsymbol{\Sigma}, \boldsymbol{\Sigma}_{u}, \boldsymbol{\Sigma}_{v}$ and $\boldsymbol{\Sigma}_{w}$, and the sparse regression parameter $\boldsymbol{\beta}$.

For the time varying parameter, we give a prior $\boldsymbol{\alpha}_{1} \sim \mathcal{N}(\boldsymbol{a}, \boldsymbol{P})$ with $\boldsymbol{a}$ is the mean and $\boldsymbol{P}$ is the covariance matrix. For the covariance matrices of the errors, we choose priors as follows:

$$
\begin{aligned}
\boldsymbol{\Sigma}^{-1} \sim W_{\mathcal{G}}(\nu, \boldsymbol{H}), & \boldsymbol{\Sigma}_{u}^{-1} \sim W_{\mathcal{G}}\left(\nu, k_{1}^{2}(n+1) \boldsymbol{H}\right), \\
\boldsymbol{\Sigma}_{v}^{-1} \sim W_{\mathcal{G}}\left(\nu, k_{2}^{2}(n+1) \boldsymbol{H}\right), & \boldsymbol{\Sigma}_{w}^{-1} \sim W_{\mathcal{G}}\left(\nu, k_{3}^{2}(n+1) \boldsymbol{H}\right),
\end{aligned}
$$

where $W_{\mathcal{G}}$ stands for a $\mathcal{G}$-Wishart distribution. For the stationarity constraint parameter $\boldsymbol{D}$, we choose a conjugate prior $\boldsymbol{D} \sim \mathcal{N}\left(\mathbf{0}, \boldsymbol{I}_{n}\right)$.

Putting a prior on the stationarity constraint matrix of a univariate $\operatorname{AR}(1)$ process is straightfoward. However, for the $\operatorname{VAR}(1)$ process in (7), the stationarity matrix $\boldsymbol{\Phi}$ has to meet the Schur-stability constraint (Roy et al., 2016), that is, it needs to satisfy $\left|\lambda_{j}(\boldsymbol{\Phi})\right|<1, j=1, \ldots, n$, where $\lambda_{j}$ stands for the $j$ th eigenvalue. Thus the parameter space of $\boldsymbol{\Phi}$ is given by

$$
\mathfrak{S}^{n}=\left\{\boldsymbol{\Phi} \in \mathbb{R}^{n \times n}:\left|\lambda_{j}(\boldsymbol{\Phi})\right|<1, j=1, \ldots, n\right\} .
$$

Clearly simply putting a conjugate matrix-normal prior on $\boldsymbol{\Phi}$ does not guarantee that all the sample draws are Schur-stable. We follow Roy et al. (2016)'s method of putting priors on $\boldsymbol{\Phi}$ through a representation as given below.

We first denote $\widetilde{\boldsymbol{\tau}}_{t}=\boldsymbol{\tau}_{t}-\boldsymbol{D}$, then the Yule-Walker equation for $\widetilde{\boldsymbol{\tau}}_{t}$ is

$$
\boldsymbol{U}=\boldsymbol{\Phi} \boldsymbol{U} \boldsymbol{\Phi}^{\prime}+\boldsymbol{\Sigma}_{v},
$$

where $\boldsymbol{U}=\mathbb{E}\left(\widetilde{\boldsymbol{\tau}}_{t} \widetilde{\boldsymbol{\tau}}_{t}^{\prime}\right)$ is a symmetric matrix. Letting $f(\boldsymbol{\Phi}, \boldsymbol{U})=\boldsymbol{U}-\boldsymbol{\Phi} \boldsymbol{U} \boldsymbol{\Phi}^{\prime}$, we have that $f(\boldsymbol{\Phi}, \boldsymbol{U})$ is a positive definite matrix if and only if $\boldsymbol{\Phi} \in \mathfrak{S}^{n}$ (Stein, 1952). Furthermore, we have the following proposition: 
Proposition 1. [Roy et al. (2016)] Given a positive definite matrix $\boldsymbol{M}$, there exists a positive matrix $\boldsymbol{U}$, and a square matrix $\boldsymbol{\Phi} \in \mathfrak{S}^{n}$ such that $f(\boldsymbol{\Phi}, \boldsymbol{U})=\boldsymbol{M}$ if and only if $\boldsymbol{U} \geq \boldsymbol{M}$ and $\boldsymbol{\Phi}=(\boldsymbol{U}-\boldsymbol{M})^{1 / 2} \boldsymbol{O} \boldsymbol{U}^{-1 / 2}$ for an orthogonal matrix $\boldsymbol{O}$ with rank $r=\operatorname{rank}(\boldsymbol{U}-\boldsymbol{M})$, where $(\boldsymbol{U}-\boldsymbol{M})^{1 / 2}$ and $\boldsymbol{U}^{-1 / 2}$ are full column rank square root of matrices $(\boldsymbol{U}-\boldsymbol{M})$ and $\boldsymbol{U}^{-1}$.

In view of Proposition 1 , given $\boldsymbol{\Phi} \in \mathfrak{S}^{n}$ and an arbitrary value of $\boldsymbol{M}$, the solution for $\boldsymbol{U}$ in (13) is given by

$$
\operatorname{vec}(\boldsymbol{U})=\left(\boldsymbol{I}_{n^{2}}-\boldsymbol{\Phi} \otimes \boldsymbol{\Phi}\right)^{-1} \operatorname{vec}(\boldsymbol{M}) .
$$

Letting $\boldsymbol{V}=\boldsymbol{U}-\boldsymbol{M}$, we have $\boldsymbol{\Phi}=\boldsymbol{V}^{1 / 2} \boldsymbol{O} \boldsymbol{U}^{-1 / 2}$, where $\boldsymbol{V}$ is a positive definite matrix, and $\boldsymbol{O}$ is an orthogonal matrix. The matrix $\boldsymbol{V}$ can be represented by the Cholesky decomposition $\boldsymbol{V}=\boldsymbol{L} \boldsymbol{\Lambda} \boldsymbol{L}^{\prime}$, where $\boldsymbol{L}$ is a lower triangular matrix and $\boldsymbol{\Lambda}$ is a diagonal matrix with positive entries. Thus the number of unknown parameters in $\boldsymbol{V}$ reduces to $n(n-1) / 2+n$. The parameter $\boldsymbol{O}$ can be decomposed by using the Cayley representation

$$
\boldsymbol{O}=\boldsymbol{E}_{\iota} \cdot\left[\left(\boldsymbol{I}_{n}-\boldsymbol{G}\right)\left(\boldsymbol{I}_{n}+\boldsymbol{G}\right)^{-1}\right]^{2}
$$

with $\boldsymbol{E}_{\iota}=\boldsymbol{I}_{n}-2 \iota \boldsymbol{e}_{1} \boldsymbol{e}_{1}^{\prime}, \iota \in\{0,1\}$ and $\boldsymbol{e}_{1}=(1,0, \ldots, 0)^{\prime}$, where $\boldsymbol{G}$ is a skew-symmetric matrix. Thus the number of parameters in $\boldsymbol{O}$ is $n(n-1) / 2+1$. By taking the logtransform, the parameters in $\boldsymbol{\Lambda}$ can be made free of restrictions. Therefore there are $n^{2}$ unrestricted parameters in $\boldsymbol{\Phi}$ plus one binary parameter. We put normal priors on the $n^{2}$ unrestricted parameters: the lower triangular elements of $\boldsymbol{L}$, the log-transformed diagonal elements of $\boldsymbol{\Lambda}$ and the lower triangular elements of $\boldsymbol{G}$. For convenience, we choose the same normal prior for those parameters and choose a binomial prior for the binary parameter $\iota$.

For the sparse regression parameter $\boldsymbol{\beta}$, we chose a spike-and-slab prior with $\boldsymbol{\beta} \sim$ $\mathcal{N}\left(\mathbf{0}, \boldsymbol{A}_{\boldsymbol{\gamma}}\right), \boldsymbol{A}_{\boldsymbol{\gamma}}=\operatorname{diag}\left(a_{1}, \ldots, a_{p}\right)$ with $a_{i}=v_{0}\left(1-\gamma_{i}\right)+v_{1} \gamma_{i}$, where $0 \leq v_{0}<v_{1}$, diag refers to a diagonal matrix with entries as specified; $\pi(\gamma \mid \theta)=\theta^{|\gamma|}(1-\theta)^{p-|\gamma|}$ with $|\gamma|=\sum_{i=1}^{p} \gamma_{i} ; \theta \sim \operatorname{Beta}\left(\zeta_{1}, \zeta_{2}\right)$.

\section{Posterior computation}

We propose a two-stage estimation algorithm to estimate the parameters. In the first stage, we adopt a fast variable selection method to obtain a point estimator for $\boldsymbol{\beta}$. In the second stage, we plug-in its estimated value and sample the remaining parameters using an MCMC algorithm.

To conduct the variable selection on $\boldsymbol{\beta}$, a popular choice would be using a SSVS method (George and McCulloch, 1993). The algorithm searches for $2^{p}$ possible combinations of $\beta_{i}$ in $\boldsymbol{\beta}$ using Gibbs sampling under $\gamma=0$ and $\gamma=1, i=1, \ldots, p$. In the multivariate setting, this method is computationally very challenging when $p$ is large. An alternative way is to use the EMVS method (Ročková and George, 2014). This method uses the EM algorithm to maximize the posterior of $\boldsymbol{\beta}$ and thus obtain the estimated model. It is computationally much faster than the SSVS method. Although SSVS gives a fully Bayesian method quantifying the uncertainty of variable selection 
through posterior distributions, the approach is not scalable for our application which involves a large sized data. Since quantifying uncertainty of variable selection is not an essential goal, as variable selection is only an auxiliary tool here to aid inference, the faster EMVS algorithm seems to be a pragmatic method to use in our application.

After obtaining $\hat{\boldsymbol{\beta}}$, we plug it into (10)-(11) and deduct $\boldsymbol{X}_{t} \hat{\boldsymbol{\beta}}$ from $\boldsymbol{Y}_{t}$. We denote the new data as $\tilde{\boldsymbol{Y}}_{t}$, and will work with the following model:

$$
\begin{aligned}
\tilde{\boldsymbol{Y}}_{t} & =\boldsymbol{z} \boldsymbol{\alpha}_{t}+\boldsymbol{\epsilon}_{t}, \\
\boldsymbol{\alpha}_{t+1} & =\boldsymbol{c}+\boldsymbol{T} \boldsymbol{\alpha}_{t}+\boldsymbol{R} \boldsymbol{\eta}_{t} .
\end{aligned}
$$

In the MCMC step, we sample the parameters in the Model (16) from their corresponding posteriors. Those parameters include: the time-varying parameters $\boldsymbol{\alpha}_{1: T}$, the stationarity constraint parameters $\boldsymbol{D}$ and $\boldsymbol{\Phi}$, the covariance matrices of the residuals $\boldsymbol{\Sigma}^{-1}, \boldsymbol{\Sigma}_{u}^{-1}, \boldsymbol{\Sigma}_{v}^{-1}$, and $\boldsymbol{\Sigma}_{w}^{-1}$.

The details of the algorithm are presented in the supplementary material (Ning et al., 2019a).

The proposed two-stage estimation algorithm is thus summarized as follows:

Stage 1: EMVS step. Choose initial values for $\boldsymbol{\beta}^{(0)}, \boldsymbol{a}_{1}^{*(0)}$ and $\boldsymbol{P}_{1}^{*(0)}$ using the revised EMVS algorithm to find the optimized value for $\boldsymbol{\beta}$.

Stage 2: MCMC step. Given $\tilde{\boldsymbol{Y}}_{t}$, we sample parameters using MCMC with the following steps:

(a) Generate $\boldsymbol{\alpha}_{t}$ using the Kalman filter and simulation smoother method.

(b) Generate $\boldsymbol{\Phi}$ using the Metropolis-Hastings algorithm.

(c) Generate $\boldsymbol{D}$.

(d) Generate covariance matrices from their respective $\mathcal{G}$-Wishart posterior densities.

(e) Go to Step (a) and repeat until the chain converges.

Skip Step (b) and (c) if no stationarity restriction is imposed on $\boldsymbol{\tau}_{t}$.

\section{A new method to infer causality}

In this section, we will introduce our new method to infer causality (in short, "the new method") along a commonly used method.

Recall the treatment effects of the commonly used method is defined in (1). Since $\sum_{t=T+1}^{T+P} \boldsymbol{Y}_{t}^{\text {cf }}$ is an unobserved quantity, we replace it by its posterior samples from $p\left(\sum_{t=T+1}^{T+P} \boldsymbol{Y}_{t}^{\mathrm{cf}} \mid \boldsymbol{Y}_{1: T}^{\mathrm{obs}}, \boldsymbol{X}_{1: T+P}\right)$.

The commonly used method may fail to detect even for a moderately sized impact for two reasons. First, the prediction intervals increase linearly as the time lag increases. 
Secondly, the trends are the only latent variables would give a response to an impact, including the random noise and the seasonality components would inflate the uncertainty of the estimated effect. For the data have a low signal-to-noise ratio, this method is which even harder to detect causal impacts.

We thus propose a new method by comparing only the posterior distributions of the latent trend in the model given the observations and the data from counterfactuals. The new method consists the following five steps:

Step 1: Applying the two-stage algorithm to obtain posterior samples for parameters in the model using the data from the period without causal impacts.

Step 2: Based on those posterior samples, obtaining sample draws of $\boldsymbol{Y}_{T+1: T+P}^{\mathrm{cf}}$ from its predictive posterior distribution $p\left(\boldsymbol{Y}_{T+1: T+P}^{\text {cf }} \mid \boldsymbol{Y}_{1: T}^{\text {obs }}, \boldsymbol{X}_{1: T+P}\right)$.

Step 3: Generating $k$ different datasets from counterfactual potential outcomes (in short, "counterfactual datasets") from the predictive posterior distribution, for the $j$-th dataset, $j \in\{1, \ldots, k\}$, denoted by $\boldsymbol{Y}_{T+1: T+P}^{\mathrm{cf}(j)}$. Then fitting each $\boldsymbol{Y}_{T+1: T+P}^{\mathrm{cf}(j)}$ into the model to obtain sample draws of the trend from its posterior distribution, which is shown in (3) (here, we replace $\boldsymbol{Y}_{T+1: T+P}^{\mathrm{cf}}$ with $\boldsymbol{Y}_{T+1: T+P}^{\mathrm{cf}(j)}$ ). Also, fitting the observed data $\boldsymbol{Y}_{1: T+P}^{\text {obs }}$ into the model and sampling from (2).

Step 4: Using the one-sided Kolmogorov-Smirnov (KS) distance to quantify the difference between the posterior distributions of the trend given by the observed data and the counterfactual datasets. The posterior distribution of the trend given by the counterfactual datasets is obtained by stacking the sample draws estimated from all the $k$ simulated datasets. Then calculating the KS distance between the two posterior distributions for each store as follows:

$$
\begin{gathered}
\sup _{x}\left[\frac{1}{k} \sum_{j=1}^{k}\left(\mathcal{F}\left(\sum_{t=T+1}^{T+P} \mu_{i t} \leq x \mid \boldsymbol{Y}_{1: T}^{\mathrm{obs}}, \boldsymbol{Y}_{T+1: T+P}^{\mathrm{cf}(j)}, \boldsymbol{X}_{1: T+P}\right)\right)\right. \\
\left.-\mathcal{F}\left(\sum_{t=T+1}^{T+P} \mu_{i t} \leq x \mid \boldsymbol{Y}_{1: T+P}^{\mathrm{obs}}, \boldsymbol{X}_{1: T+P}\right)\right]
\end{gathered}
$$

where $i=1, \ldots, n$, and $\mathcal{F}(\cdot)$ stands for the empirical distribution function of the obtained MCMC samples.

Step 5: Calculating the $k \times(k-1)$ pairwise one-sided KS distances between the posterior distributions of the trends given by the $k$ simulated counterfactual datasets, that is to calculate the following expression

$$
\begin{array}{r}
\sup _{x}\left[\mathcal{F}\left(\sum_{t=T+1}^{T+P} \mu_{i t} \leq x \mid \boldsymbol{Y}_{1: T}^{\mathrm{obs}}, \boldsymbol{Y}_{T+1: T+P}^{\mathrm{cf}(j)}, \boldsymbol{X}_{1: T+P}\right)\right. \\
\left.-\mathcal{F}\left(\sum_{t=T+1}^{T+P} \mu_{i t} \leq x \mid \boldsymbol{Y}_{1: T}^{\mathrm{obs}}, \boldsymbol{Y}_{T+1: T+P}^{\mathrm{cf}\left(j^{\prime}\right)}, \boldsymbol{X}_{1: T+P}\right)\right]
\end{array}
$$

where $j, j^{\prime}=1, \ldots, k, j \neq j^{\prime}$. Then, for each $i$, choosing the $95 \%$ upper percentile among those distances as a threshold to decide whether the KS distance calculated 
from (17) is significant or not. If the KS distance is smaller than this threshold, then the corresponding causal impact is declared not significant.

The use of a threshold is necessary, since the two posterior distributions of the trend obtained under observed data and the data from the counterfactual are not exactly equal even when there is no causal impact. Our method automatically selects a data-driven threshold through a limited repeated sampling as in multiple imputations.

So far we described the commonly used method and the new method in the setting where the period without a causal impact comes before that with the impact. However, the new method can be extended to allow datasets in more general situations when: 1) there are missing data from the period without causal impact; 2) the period without causal impact comes after the period with a impact; 3 ) there are more than one periods without causal impact, both before and after the period with a impact. This is because the KFBS method is flexible to impute missing values at any positions in a times series dataset.

\section{Simulation study}

In this section, we conduct a simulation study to compare the two different methods introduced in the last section. To keep the analysis simple, we only consider the setting that the period with causal impact follows that without the impact. We also conduct convergence diagnostics for MCMC chains and a sensitivity analysis for the new method, the results are shown in Section 4 of the supplementary material (Ning et al., 2019a).

\subsection{Data generation and Bayesian estimation}

We simulate five spatially correlated datasets, and assume the precision matrices in the model have the adjacency matrix as follows:

$$
\left(\begin{array}{lllll}
1 & 1 & 0 & 0 & 0 \\
1 & 1 & 1 & 0 & 0 \\
0 & 1 & 1 & 1 & 0 \\
0 & 0 & 1 & 1 & 1 \\
0 & 0 & 0 & 1 & 1
\end{array}\right),
$$

that is, we assume variables align in a line with each one only correlated with its nearest neighbors. We generate daily time series for an arbitrary date range from January 1, 2016 to April 9, 2016, with a perturbation beginning on March 21, 2016. We specify dates in the simulation to facilitate the description of the intervention period. We first generate five multivariate datasets for test stores with varying levels of impact and label them as Datasets $1-5$.

For each Dataset $i, i=1, \ldots, 5$, the trend is generated from $\mu_{i t} \sim \mathcal{N}\left(0.8 \mu_{i, t-1}, 0.1^{2}\right)$ with $\mu_{i 0}=1$. The weekly components are generated from two sinusoids of the same frequency 7 as follows:

$$
\delta_{i t}=0.1 \times \cos (2 \pi t / 7)+0.1 \times \sin (2 \pi t / 7) .
$$


Additional datasets for 10 control stores are generated, each from an $\operatorname{AR}(1)$ process with coefficient 0.6 and standard error 1 . We let the first and second datasets to have regression coefficients $\beta_{1}=1, \beta_{2}=2$ and let the rest to be 0 . We then generate the residuals $\boldsymbol{\epsilon}_{t}$ in the observation equation from the multivariate normal distribution $\mathcal{N}(\mathbf{0}, \boldsymbol{\Sigma})$ with precision matrix having sparsity structure given by (19). We set the diagonal elements for $\boldsymbol{\Sigma}^{-1}$ to 10 , and its non-zero off-diagonal elements to 5 . The simulated data for test stores are the sum of the simulated values of $\boldsymbol{\mu}_{t}, \boldsymbol{\delta}_{t}, \boldsymbol{X}_{t} \boldsymbol{\beta}$ and $\boldsymbol{\epsilon}_{t}$. The causal impacts are generated as follows: for each Dataset $i, i=1, \ldots, 5$, we add an impact scale $\frac{(i-1)}{2} \times(\log 1, \ldots, \log 20)$ from March 21, 2016 to April 9, 2016. Clearly no causal impact is added in Dataset 1.

We impose the graphical structure with adjacency matrix in (19) in both observed and hidden processes in the model and then apply the two-stage algorithm to estimate parameters. In Stage 1, we apply the revised EMVS algorithm. We choose the initial values $\boldsymbol{\beta}^{(0)}$ and $\boldsymbol{a}_{1}^{*(0)}$ to be the zero vectors and the first $15 \times 15$ elements of $\boldsymbol{P}_{1}^{*(0)}$, which correspond to the covariances of the trend, local trend and seasonality components, to be a diagonal matrix. The remaining elements in $\boldsymbol{P}_{1}^{*(0)}$ are set to 0 . We select 20 equally spaced relatively small values for $v_{0}$ from $10^{-6}$ to 0.02 and a relatively larger value for $v_{1}, 10$. For the prior of $\theta$, we set $\zeta_{1}=\zeta_{2}=1$. The maximum number of iterations of the EMVS algorithm is chosen to be 50 . We calculate the threshold of non-zero value of $\boldsymbol{\beta}_{i}$ from the inequality: $p\left(\gamma_{i}=1 \mid \beta_{i}, \boldsymbol{Y}_{t}^{*}, \boldsymbol{X}_{t}^{*}\right)>0.5$ (See the detailed discussions in Ročková and George, 2014). Then the threshold can be expressed as

$$
\left|\beta_{i}^{\text {th }}\right| \geq \sqrt{\frac{\log \left(v_{0} / v_{1}\right)+2 \log (\hat{\theta} /(1-\hat{\theta}))}{v_{1}^{-1}-v_{0}^{-1}}},
$$

where $\hat{\theta}$ is the maximized value obtained from the EMVS algorithm. Ročková and George (2014) also suggested using a deterministic annealing variant of the EMVS (DAEMVS) algorithm which maximizes

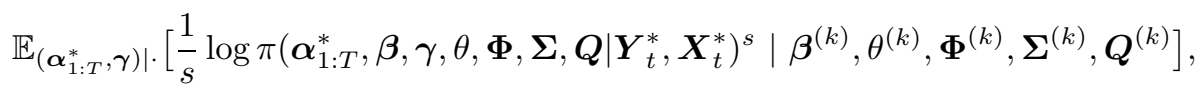

where $0 \leq s \leq 1$. The parameter $1 / s$ is known as a temperature function (Ueda and Nakano, 1998). When the temperature is higher, that is when $s \rightarrow 0$, the DAEMVS algorithm has a higher chance to find a global mode and thus reduces the chance of getting trapped at a local maximum.

Figure 2 compares the results for using EMVS and DAEMVS with $s=0.1$ algorithms. We plot $\hat{\boldsymbol{\beta}}$ and their thresholds based on 20 different values of $v_{0}$ from $10^{-6}$ to 0.02 . From the plot, the estimated values for $\boldsymbol{\beta}$ using both EMVS and DAEMVS methods are close to their true values.

The true zero coefficients are estimated to be very close to 0 . However, we observe that the values of $\beta_{i}^{\text {th }}$ is larger by using the EMVS method compared to the DAEMVS method. This is because in the region where $v_{0}$ is less than 0.005 , the $\hat{\theta}$ estimated from EMVS is very close to 0 , thus the negative value of $\log (\hat{\theta} /(1-\hat{\theta}))$ is very large and 
EMVS

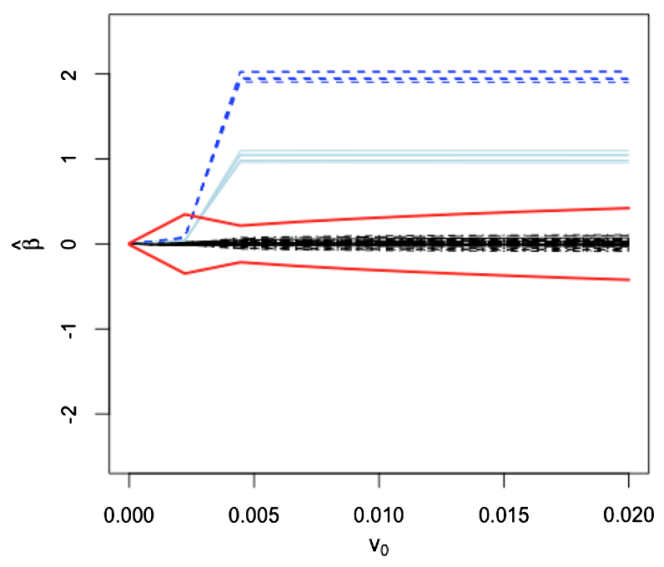

DAEMVS $(s=0.1)$

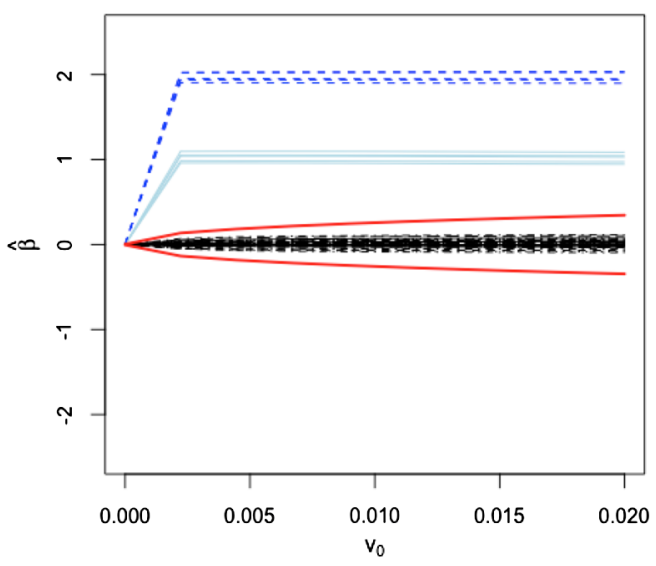

Figure 2: EMVS (left) and DAEMVS (with $s=0.1$ ) (right) estimation of $\boldsymbol{\beta}$ based on the simulated datasets. The dark blue lines are the parameters that have simulated values 2 ; the light blue lines are the parameters that have simulated values 1 and the black lines are the parameters that have simulated values 0 . The red lines are the calculated $\beta_{i}^{\text {th }}$ values, within the two red lines, the parameters should be considered as zero parameters.

the threshold becomes larger. Based on the simulation results, we use DAEMVS with $s=0.1$ throughout the rest of the paper.

The DAEMVS gives a smaller value of $\beta_{i}^{\text {th }}$, yet the thresholds can distinguish the true zero and non-zero coefficients in this case. Nevertheless it may miss a non-zero coefficient if the coefficient is within the thresholds. In practice, since our goal is to identify significant control variables and use them to build counterfactuals for a causal inference, we may choose to include more variables than the threshold suggests provided that the total number of included variables is still manageable.

Recall that in the Stage 1, we used a conjugate prior for $\operatorname{vec}(\boldsymbol{\Phi})$ instead of the originally proposed prior described in Section 1.3. Here, we want to make sure the change of prior would not affect the results of $\hat{\boldsymbol{\beta}}$ too much. We conduct the analysis by choosing two different values of the covariance matrix of the prior: $\boldsymbol{I}_{5}$ and $0.01 \times \boldsymbol{I}_{5}$. We found the estimates $\hat{\boldsymbol{\beta}}_{\text {s }}$ are almost identical to the estimated values shown in Figure 2. We also consider using other two models: one ignores the stationarity constraint for $\boldsymbol{\tau}_{t}$ (henceforth the "nonstationary model"); another ignores the time dependency of the model (henceforth the "misspecified model"). To be more explicit, for the nonstaionary model, we let the local linear trend follow (8). The misspecified model is given by $\boldsymbol{Y}_{t}^{*}=\boldsymbol{X}_{t}^{*} \boldsymbol{\beta}+\boldsymbol{\varsigma}_{t}$, with $\boldsymbol{\varsigma}_{t}$ s are i.i.d random errors with multivariate normally distributed and mean 0 by ignoring their dependency. We conduct DAEMVS with $s=0.1$ for both of the two models. In the nonstationary model, we choose a diffuse prior for $\boldsymbol{\alpha}_{1}^{*}$ and change the covariance corresponding to the local linear trend in $\boldsymbol{P}_{1}^{*(0)}$ to be $10^{6} \times \boldsymbol{I}_{5}$. In the misspecified model, the M-step can be simplified to only updates for $\boldsymbol{\beta}, \theta$ and the covariance matrix of $\boldsymbol{\varsigma}_{t}$. We plot the results into Figure 3. Comparing the results 

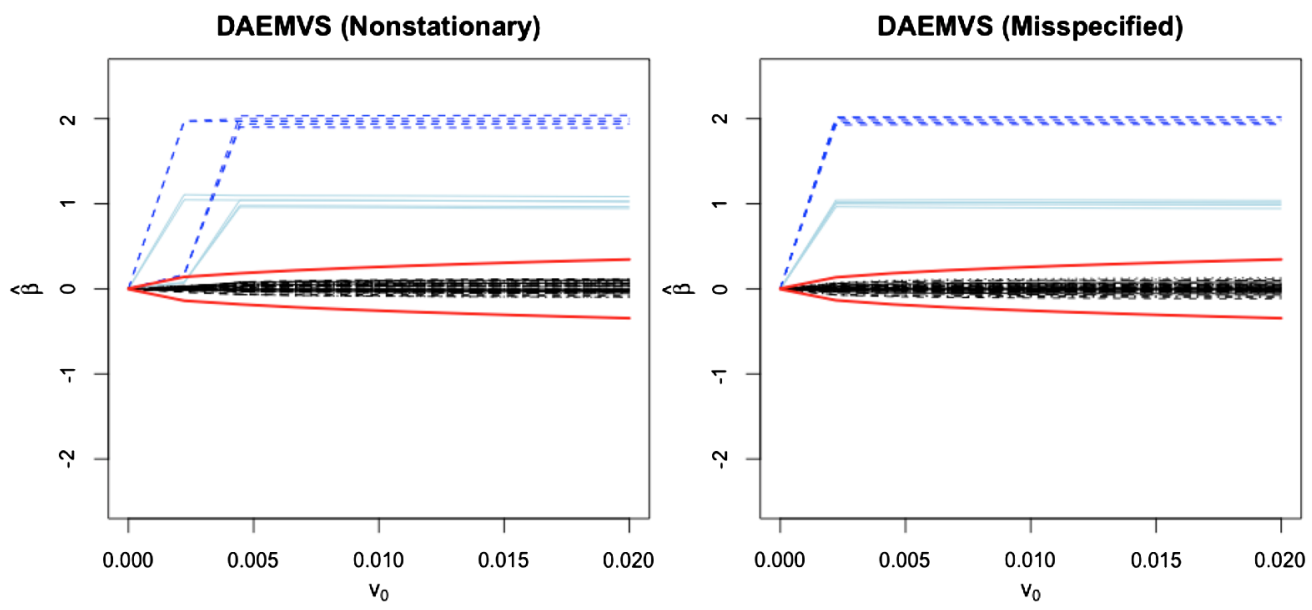

Figure 3: DAEMVS (with $s=0.1$ ) estimation of $\boldsymbol{\beta}$ based on the simulated datasets using the nonstationary model (left) and the misspecified model (right). The dark blue lines are the parameters that have simulated values 2 ; the light blue lines are the parameters that have simulated values 1 and the black lines are the parameters that have simulated values 0 . The red lines are the calculated $\beta_{i}^{\text {th }}$ values, within the two red lines, the parameters should be considered as zero.

in Figure 3 with Figure 2, there are not much differences among the results obtained using the three different models for estimating $\boldsymbol{\beta}$.

In Stage 2, we plug-in $\hat{\boldsymbol{\beta}}$ and calculate $\tilde{\boldsymbol{Y}}_{t}$ in (16). We choose the prior for the rest of parameters as follows: we let $\boldsymbol{\alpha}_{1} \sim \mathcal{N}(\mathbf{0}, \boldsymbol{I})$. If $\boldsymbol{\tau}_{t}$ is a nonstationarity process, the initial condition is considered as a diffuse random variable with large variance (Durbin and Koopman, 2002). Then we let the covariance matrix of $\boldsymbol{\tau}_{t}$ to be $10^{6} \times \boldsymbol{I}_{5}$. We let $\nu=1, k_{1}=k_{2}=k_{3}=0.1$. We choose $\boldsymbol{H}=\boldsymbol{I}_{5}$ and the priors for 25 parameters decomposed from $\boldsymbol{\Phi}$ to be $\mathcal{N}\left(0, \sqrt{5}^{2}\right)$, and let $\iota \sim \operatorname{Bernoulli}(0.5)$. We run total 10,000 MCMC iterations with the first 2,000 draws as burn-in. An MCMC convergence diagnostic and a sensitive analysis of the model are conducted, we include their results in the supplementary file.

\subsection{Performance of the commonly used causal inference method}

In this section, we study the performance of the commonly used method. The causal effect is estimated by taking the difference between observed data during causal period and the potential outcomes of counterfactuals during that period. In Stage 1, we use the DAEMVS $(s=0.1)$ algorithm to estimate $\hat{\boldsymbol{\beta}}$ for the model (10)-(11). A stationarity constraint is added on the local linear trend $\boldsymbol{\tau}_{t}$. In Stage 2, we consider two different settings for $\tau_{t}$ : with and without adding the stationarity constraint. We choose Dataset 4 as an example and plot accuracy of the model based on the two different settings in Figure 4. There are four subplots: the left two subplots are the results for the model 


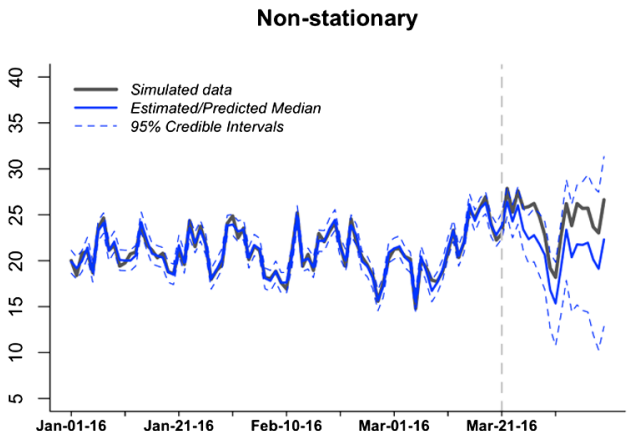

(a)

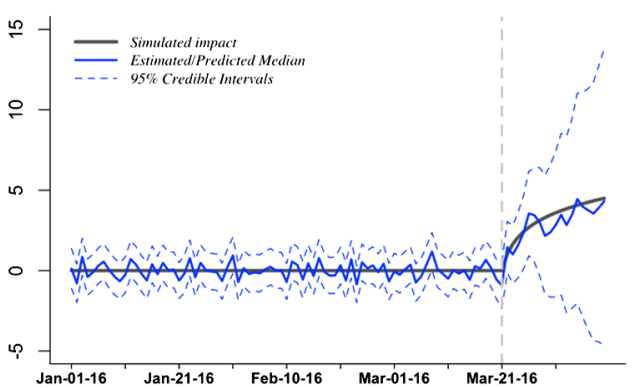

(b)

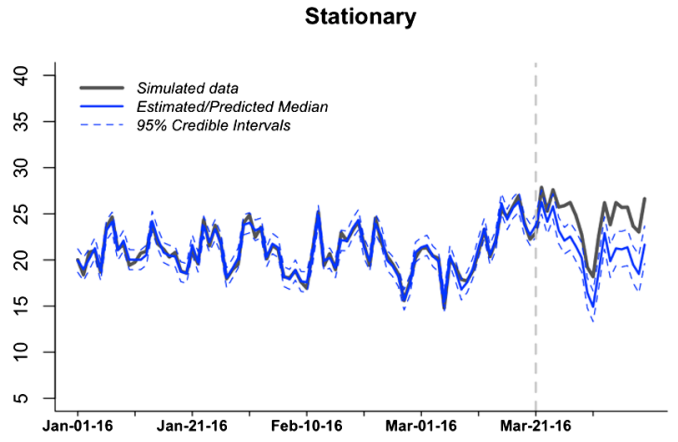

(c)

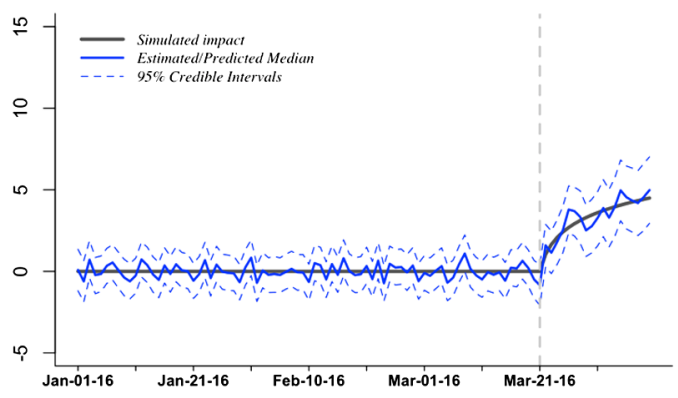

(d)

Figure 4: Plot of the causal impact in Dataset 4 using models with a stationary and a nonstationary local linear trend. (a) and (c) are the plots of estimation (before March 21, 2016) and prediction (after March 21, 2016) of Dataset 4 without stationarity constraint (left) and with stationarity constraint (right). The gray line is the simulated dataset, the blue line is the estimated posterior median of the dataset using the model, the dashed blue line is the corresponding $95 \%$ credible and prediction intervals. (b) and (d) are the plots of estimated causal impact by taking the difference between the observed data and Bayesian estimates using the model with a nonstationary local linear trend (left) and the model with a stationary local linear trend (right). The black line is the simulated true impact, the blue line is the estimated median of the impact, the dashed blue lines are the corresponding $95 \%$ credible and prediction intervals.

with a nonstationary local linear trend and the right two subplots are the results for the model with a stationary local linear trend. Before the period with a causal impact, which is March, 21, 2016, the estimated posterior medians and 95\% credible intervals obtained from the two models are close (see plots (b) and (d) in Figure 4); but their prediction intervals during the period with a causal impact are quite different. In the model with a nonstationary local linear trend, the prediction intervals are much wider and expand more rapidly than those resulting from the model with a stationary local linear trend. In the former case, the observed data during the campaign are fully contained inside the prediction intervals and thus failed to detect a causal impact. However, the model with 


\begin{tabular}{lccc}
\hline & Simulated impact & Nonstationary & Stationary \\
\hline Dataset 1 & 0.00 & $0.00[-4.419,4.425]$ & $0.29[-1.440,1.996]$ \\
Dataset 2 & 1.06 & $0.64[-3.989,5.298]$ & $1.07[-0.648,2.780]$ \\
Dataset 3 & 2.12 & $1.22[-3.758,5.965]$ & $2.27[0.399,4.014]$ \\
Dataset 4 & 3.18 & $2.83[-1.793,7.575]$ & $3.16[1.500,4.862]$ \\
Dataset 5 & 4.23 & $4.25[-0.249,8.771]$ & $4.25[2.520,5.904]$ \\
\hline
\end{tabular}

Table 1: Posterior medians and 95\% credible intervals of average causal impacts for simulated datasets estimated using the multivariate models with a stationary and a nonstationary local linear trend.

a stationary local linear trend gives only moderately increasing prediction intervals and thus can detect the causal impact. Plots (b) and (d) shown in the bottom of Figure 4 are the estimated causal impact in each model for Dataset 4 calculated by taking the difference between observed values and counterfacutal potential outcomes. In each plot, the estimated causal impact medians are able to capture the shape of the simulated causal impact. However, the prediction intervals in plot (b) contain the value 0 and thus negate the impact. The shorter prediction intervals in plot (d) do not contain the value 0 , and thus indicate the existence of a impact.

To give an overall picture of the model fitting for the five simulated datasets, we summarize the posterior medians and their $95 \%$ credible intervals of the estimated causal impact for all the datasets in Table 1. In the model with a nonstationary local linear trend, no impacts are detected for all the five datasets since their corresponding prediction intervals all contain the value 0 . In the model with a stationary local linear trend on $\boldsymbol{\tau}_{t}$, the impacts are successfully detected for the last three datasets. For Dataset 2, it has a weaker impact. Its impact is not detected even after imposing the stationarity constraint. Also, when the stationarity constraint is imposed, including the intercept $\boldsymbol{D}$ in (7) helps give a robust long run prediction. Thus, from Table 1, we find that the estimated medians using the model with a stationary local linear trend are closer to the true impact compared with that obtained from using the model with a nonstationary local linear trend.

In the setting where the sales in the test stores are spatially correlated, the use of the multivariate model with a stationary local linear trend is necessary for obtaining more accurate estimates for causal effects. We compare the results with a univariate model which ignores the correlation between the five simulated datasets. We fit the five datasets independently into that model. The model is the univariate version of the model (10)-(11). In the univariate model, the errors $\boldsymbol{\epsilon}_{t}, \boldsymbol{u}_{t}, \boldsymbol{v}_{t}$ and $\boldsymbol{w}_{t}$ become scalars. We denote $\sigma^{2}, \sigma_{u}^{2}, \sigma_{v}^{2}$ and $\sigma_{w}^{2}$ as their corresponding variances. We choose their priors as $\sigma^{-2} \sim \operatorname{Gamma}\left(\frac{0.1}{2}, \frac{0.1 \times \mathrm{SS}}{2}\right), \sigma_{u}^{-2}, \sigma_{v}^{-2}, \sigma_{w}^{-2} \sim \operatorname{Gamma}(0.01,0.01 \times \mathrm{SS})$, where $\mathrm{SS}=\sum_{t=1}^{T}\left(y_{t}-\bar{y}\right)^{2} /(T-1)$ and $\bar{y}=\sum_{t=1}^{T} y_{t} / T$. The parameters $\boldsymbol{D}$ and $\boldsymbol{\Phi}$ in (7) also become scalars and to be denoted by $d$ and $\phi$ respectively. We give them the priors $d \sim \mathcal{N}\left(0,0.1^{2}\right)$ and $\phi \sim \mathcal{N}\left(0,0.1^{2}\right) \mathbb{1}_{(-1,1)}$.

In order to make the comparison between the multivariate model and the univariate model meaningful, we plug-in the same $\hat{\boldsymbol{\beta}}$ obtained from Stage 1 for both models. We 


\begin{tabular}{ccc}
\hline & Simulated impact & Stationary (univariate) \\
\hline Dataset 1 & 0.00 & $0.17[-2.197,2.472]$ \\
Dataset 2 & 1.06 & $1.03[-1.365,3.473]$ \\
Dataset 3 & 2.12 & $2.16[-0.370,4.476]$ \\
Dataset 4 & 3.18 & $3.20[0.821,5.748]$ \\
Dataset 5 & 4.23 & $4.08[1.564,6.489]$ \\
\hline
\end{tabular}

Table 2: Posterior medians and $95 \%$ credible intervals of average causal impacts for simulated datasets estimated using the univariate model.

conduct an MCMC algorithm for the five datasets separately using the univariate model by sequentially sampling draws from the corresponding posterior distributions of $\alpha_{1: T}$, $d, \phi, \sigma^{2}, \sigma_{u}^{2}, \sigma_{v}^{2}$ and $\sigma_{w}^{2}$. We run the MCMC algorithm for 10,000 iterations and treat the first 2,000 as burn-in. The estimated causal impacts are shown in Table 2. By comparing the results with the results in Table 1, the univariate model produces wider credible intervals for all of datasets even though their posterior medians are close to the truth. Thus the multivariate model with a stationary local linear trend is more accurate for detecting a causal impact.

We conduct additional independent 10 simulation studies by generating datasets using the same scheme which described above, but using different random number generators from the software. We conduct the same analysis for the 10 simulated studies using the multivariate model with stationarity constraints. All of these studies show that the commonly used method failed to detect causal effect for the second dataset, which is the one with the smallest amount of simulated causal impact.

\subsection{Performance of the new method to infer causality}

In this section, we study the performance of the new method. We use the same simulated data in Section 6.1. We calculate the one-sided KS distance in (17) and the threshold in (18) for each $i=1, \ldots, n$. We also calculate the one-sided KS distances

$$
\begin{gathered}
\sup _{x}\left[\frac{1}{k} \sum_{j=1}^{k}\left(\mathcal{F}\left(\sum_{t=T+1}^{T+m} \mu_{i t} \leq x \mid \boldsymbol{Y}_{1: T}^{\mathrm{obs}}, \boldsymbol{Y}_{T+1: T+m}^{\mathrm{cf}(j)}, \boldsymbol{X}_{1: T+m}\right)\right)\right. \\
\left.-\mathcal{F}\left(\sum_{t=T+1}^{T+m} \mu_{i t} \leq x \mid \boldsymbol{Y}_{1: T+m}^{\mathrm{obs}}, \boldsymbol{X}_{1: T+m}\right)\right]
\end{gathered}
$$

and the corresponding thresholds for $m=1$ to $m=P$. This allows to see how the KS distances grow over time.

We plot the results in Figure 5. There are five subplots in that figure with each represents one simulated dataset. For each subplot, the red line represents the onesided KS distances between posteriors from a test store and its counterfactuals, and the lightblue line represents its corresponding thresholds. The threshold is calculated based 

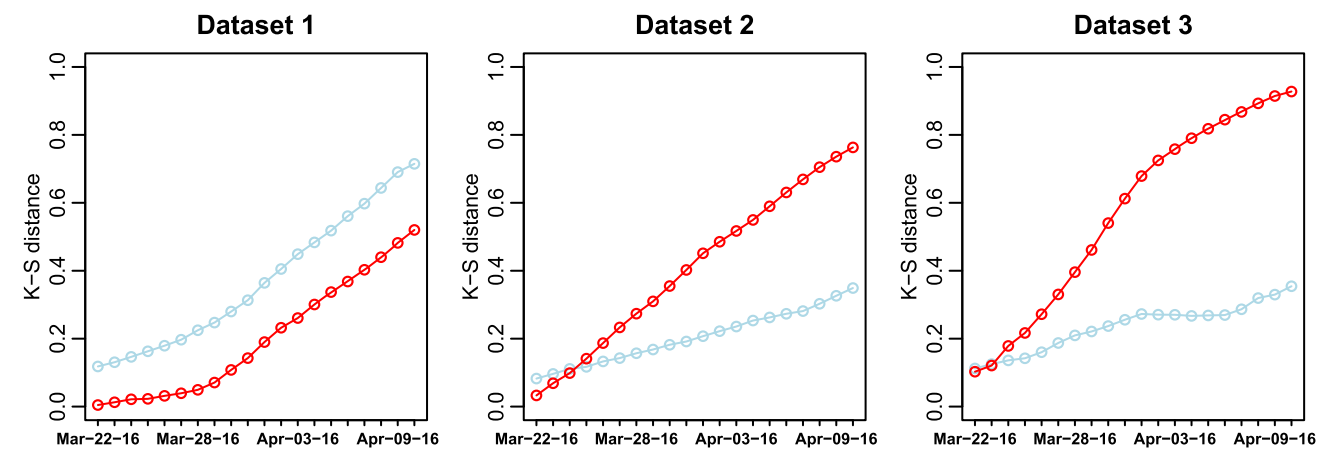

Dataset 4
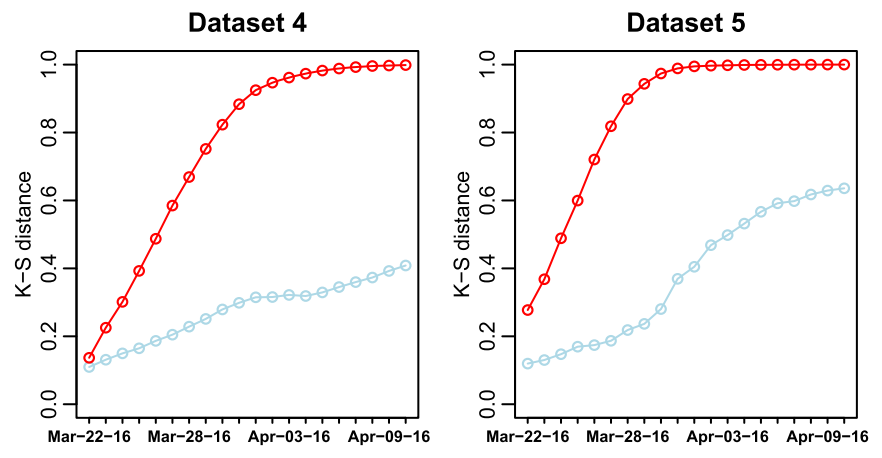

Figure 5: Results of applying the new method to detect causal impacts in Dataset 1, ..., Dataset 5 using the multivariate model with a stationary local linear trend during the causal period from March, 22, 2016 to April, 9, 2016. In each subplot, the red line gives the one-sided KS distances between two posterior distributions with one is given the data of counterfactuals; the light blue line gives the corresponding thresholds.

on $k=30$ simulated counterfactual datasets. In the plot, Dataset 1 is the only one with the one-sided KS distances completely below the thresholds and it is the dataset which does not receive any impacts. This suggests that our method has successfully distinguished between impact and no impact in these datasets. For Dataset 2, the impact at the early period is small, thus we observe the causal impact in the first three predicting periods are not significant; however, the new method can detect the impact after the fourth period.

We also summarized the results in Table 3. Compared with the results from the commonly used method (see Table 1), the new method shows a significant improvement in detecting causal impacts. From Dataset 3 to Dataset 5, the one-sided KS distances are all above their corresponding thresholds. Also, as the impact grows stronger, we observe that the distances becomes larger. The thresholds too increase along the time, since the predicting intervals for the trends become wider.

To check the performance of the new method, we conduct 10 more simulation studies using the data generated from the same model. Although the values of the one-sided 


\begin{tabular}{ccccccc}
\hline & & Dataset 1 & Dataset 2 & Dataset 3 & Dataset 4 & Dataset 5 \\
\hline March, 22 & KS distance & 0.005 & 0.033 & 0.103 & 0.137 & 0.277 \\
(1st day) & Threshold & 0.118 & 0.083 & 0.112 & 0.110 & 0.120 \\
\hline March, 31 & KS distance & 0.143 & 0.402 & 0.612 & 0.884 & 0.989 \\
(10th day) & Threshold & 0.313 & 0.192 & 0.256 & 0.299 & 0.369 \\
\hline April, 9 & KS distance & 0.520 & 0.763 & 0.928 & 0.999 & 1.000 \\
(20th day) & Threshold & 0.715 & 0.349 & 0.354 & 0.409 & 0.636 \\
\hline
\end{tabular}

Table 3: Results of the one-sided KS distances and thresholds obtained by applying the new method to detect causal impacts in Dataset $1, \ldots$, Dataset 5 using the multivariate model with a stationary local linear trend. We only present the results at the dates March 22, 2016, March 31, 2016 and April. 9, 2016 which correspond to the 1st day, 10th day and 20th day during the causal period.

KS distances and thresholds are not identical for each simulation, since the model is highly flexible and the estimated trend is sensitive to local changes of a dataset, the new method successfully detects the causal impacts in Dataset $2, \ldots$, Dataset 5 .

We applied the new method to the univariate model, which is described in Section 6.2, using the same simulated dataset. The graphical and tabular representations of the results are presented in Section 3 of the supplementary material (Ning et al., 2019a). We found that by comparing with the results obtained from the multivariate model (see Figure 5), the thresholds are much larger among all the datasets. Recall that from Table 2 , the credible intervals estimated using the commonly used method are wider. Thus when we randomly draw samples from a counterfactual with a larger variance, the posterior distributions for their trend are more apart. As a result, the pairwise one-sided KS distances between the posterior distributions of the trends are larger. Even though the thresholds are larger when using the univariate model, unlike the results obtained by using the commonly used method, the new method can still detect the causal impact for almost all the datasets which received an impact successfully, except for the very weak impacts in Dataset 2 during the first three periods and Dataset 3 during the first period.

\section{Application to a real dataset}

In this section, we present the results of a real data analysis for measuring the causal impact of an online advertising campaign (run by MaxPoint) for a consumer product at a large national retail chain.

Due to commercial confidentiality, we do not show full details of the results, but the following description explains how our method works in this real dataset. MaxPoint targets this campaign at 627 test stores and 318 control stores spread out across the country and collects weekly data throughout the campaign. We choose all the control stores in the corresponding state for each dataset. If a state does not at all have control stores, we remove such data from the analysis. In Stage 1, we use the DAEMVS (with $s=0.1$ ) algorithm to select the control stores for each test store. If for a test store, all 
the potential control stores are eliminated by the DAEMVS algorithm we also eliminate that store from the causal analysis, because without building a counterfactual, the causal inference cannot be conducted. After making the selection, we conduct the causal analysis on 323 test stores in total. For each dataset, there are 46 weekly observations in total with the last 10 observations occurring in the causal period. Since the length before the causal period is only 35 per dataset, we have to separate these 323 stores into smaller datasets and fit the model separately on them. As large national chain retailers organize promotional and operations activity differently in each state, we treat stores in different states as independent. State-wise splitting typically keeps the number of stores less than 15. If one state has more than 15 stores, we split further into subregions to meet the requirement. We further assume that the stores in two different subregions behave independently. The regions are separated based on city boundaries. Within each region, we assume that stores are connected with each other. This means that the inverse covariance matrix (equivalently, the covariance matrix) follows a block-diagonal structure with at most 15 nodes in a block.

We assume the three causal assumptions in Section 2 hold. The following table summarizes the number of stores with significant causal effects from the advertising campaign. From the table we found that the number of stores are increasing from the first week to the last week. During the first five weeks, the number of stores that received causal impact increased rapidly compared with that in the last five weeks.

\begin{tabular}{cccccc}
\hline & 1st week & 2nd week & 3rd week & 4 th week & 5 th week \\
Number of stores & 23 & 44 & 55 & 62 & 73 \\
\hline & 6 th week & 7 th week & 8 th week & 9 th week & 10 th week \\
Number of stores & 72 & 77 & 78 & 82 & 84 \\
\hline
\end{tabular}

Table 4: Number of test stores that received significant causal impacts for each week of running the advertisement campaign by using the multivariate model with a stationary local linear trend.

Not only the number of impacted stores increased during the advertising campaign period (shown in Table 4), the magnitudes of the impacts in those stores also increased. In Figure 6, we plot the estimated one-sided KS distances for stores along with their locations at Weeks 2, 5 and 10. In each figure, we only plot the stores with significant causal effects. The red dots represent the stores with the one-sided KS distances larger than their corresponding thresholds, which suggests that those stores received significant causal effects. The grey dots represent the stores that do not show significant causal effects. We find that the magnitudes of the impacts for most of the stores have a larger increase from the first five weeks compared with the last five weeks. Comparing the plots of the fifth week and the tenth week, we find that only a few stores in California, South Dakota, Ohio and Texas got increased causal effects.

We also conduct an analysis by assuming that the test stores are independent and thus ignoring their spatial correlation. Table 5 lists the number of stores that received significant causal effects. The numbers are smaller than those obtained using the multivariate model. This suggests most of the impacts are weak and the spatial correlation between sales in different stores help detect the weaker impacts. 


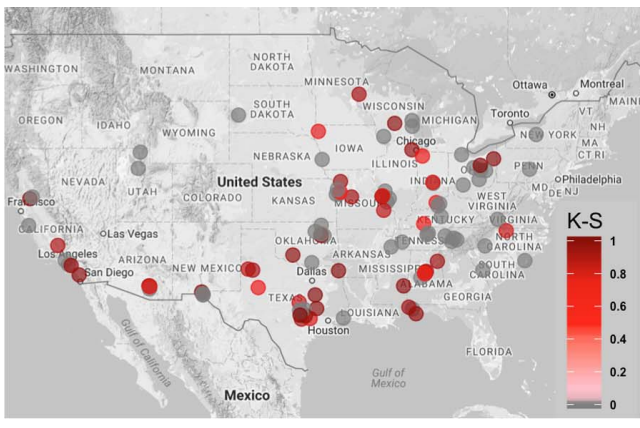

(a) 2nd week

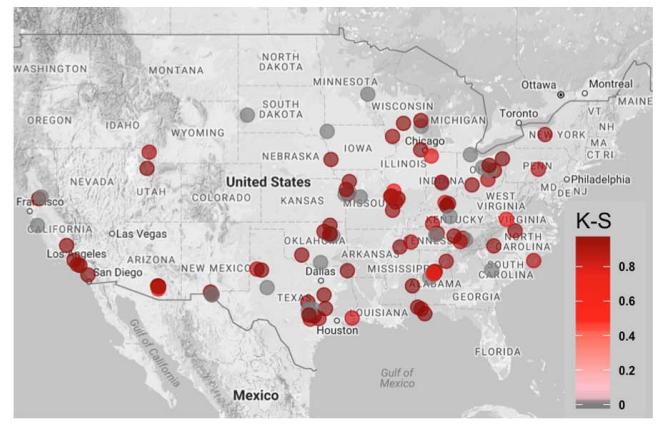

(b) 5 th week

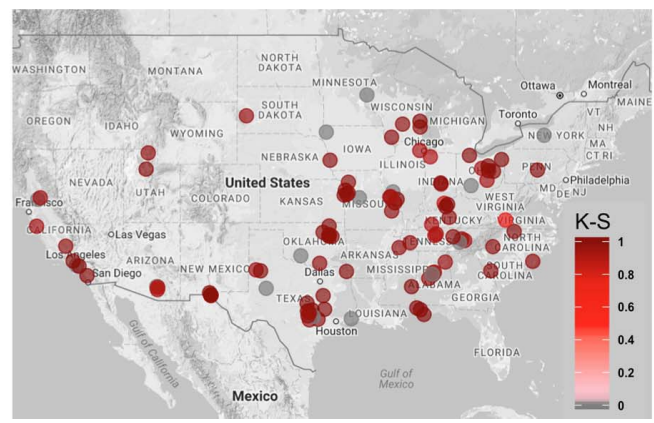

(c) 10 th week

Figure 6: Plot of the causal impacts at test stores at end of the second week (a), the fifth week (b) and the last week (c) for an advertising campaign of a consumer product at a large national retail chain. The impacts below their thresholds are set to zero. The United States map is produced using Google Maps, 2017.

\begin{tabular}{lccccc}
\hline & 1st week & 2nd week & 3rd week & 4th week & 5 th week \\
Number of stores & 25 & 19 & 22 & 23 & 18 \\
\hline & 6 th week & 7 th week & 8 th week & 9 th week & 10 th week \\
Number of stores & 17 & 15 & 15 & 13 & 14 \\
\hline
\end{tabular}

Table 5: Number of test stores that received significant causal impacts for each week of running the advertisement campaign by using the univariate model.

\section{Conclusion and discussion}

In this paper, we proposed a novel causal inference method which compares the posterior distributions of the latent trend conditional on two different sets of data: one is the observed data which contain a causal effect; the other one is the data from a synthetic control. We calculated the one-sided KS test statistics between the two posterior distributions. A threshold was used to decide whether a causal impact is significant or not. In the simulation study, we showed that our method can detect a smaller sized 
causal impact more efficiently compared with the commonly used method even when the model is slightly misspecified. The new causal inference method is not restricted to the specific structural time series model used in this paper and can be applied to many other models in different applications.

We used a multivariate structural time series model to estimate the causal impact of a stimulus on subjects such as an advertising campaign for each individual store. Sales in those stores are spatially correlated. A Bayesian analysis was used to estimate parameters in this model. We imposed sparsity on the precision matrix based on the distance between each pair of stores. The sparsity was imposed through a $\mathcal{G}$-Wishart prior, where the graph $\mathcal{G}$ can be either decomposable or non-decomposable. We restricted the hidden process $\boldsymbol{\tau}_{t}$ to be stationary in order to stabilize the prediction intervals. To sample its time-varying variables, we used the Kalman filter and simulation smoother algorithm. This algorithm can be used to impute missing values inside the MCMC loops.

We used the revised EMVS algorithm to select control stores. We also discussed the advantage of using the DAEMVS algorithm which is a modified version of the EMVS algorithm. Compared to the EMVS algorithm, the DAEMVS algorithm reduces the chance of getting trapped at a local maximum. Both the EMVS and DAEMVS algorithms are computationally much faster than the sampling based method like SSVS. Since the EMVS algorithms cannot be incorporated into MCMC loops, we proposed a two-stage algorithm to estimate parameters. In Stage 1, we used the DAEMVS to obtain $\hat{\boldsymbol{\beta}}$; in Stage 2, we plugged-in $\hat{\boldsymbol{\beta}}$ and used an MCMC algorithm to obtain posterior distributions of the remaining parameters.

We compared the multivariate model with the univariate model which assumes independence between responses based on simulated datasets. The results indicate that the univariate model gives wider credible intervals (if using the commonly used method) and larger threshold (if using the new method) than the multivariate model. Thus incorporating of the spatial relationships between test stores is beneficial.

Finally, we analyzed a real dataset on sales data of products distributed through brick and mortar retail stores for an advertising campaign run by MaxPoint. Even though, due to commercial confidentiality, we did not provide the full details of the results, the summarization tables of the number of stores that received significant impact suggests the effectiveness of using the new causal inference method.

\section{Supplementary Material}

Supplement to "Bayesian method for causal inference in spatially-correlated multivariate time series" (DOI: 10.1214/18-BA1102SUPPA; .pdf). This supplementary material contains five sections. Sections 1 and 2 provide the details on deriving the two-stage algorithm and the revised EMVS algorithm. Section 3 provides graphical and tabular representations of the results of the new method to infer causality using the univariate model. Section 4 provides model checking results. Section 5 describes the Kalman filter and backward smoothing algorithm. 
$\mathrm{R}$ code for "Bayesian method for causal inference in spatially-correlated multivariate time series" (DOI: 10.1214/18-BA1102SUPPB; .zip). This supplementary material includes the original Bayesian multivariate time series model code written in R (Ning et al., 2019b). The code is also available on the website: https://github.com/Bo-Ning/ Bayesian-multivariate-time-series-causal-inference.

\section{References}

Abadie, A. (2005). Semiparametric difference-in-differences estimators. The Review of Economic Studies 72, 1-19. MR2116973. doi: https://doi.org/10.1111/00346527.00321 .5

Abadie, A. and J. Gardeazabal (2003). The economic costs of conflict: A case study of the basque country. American Economics Review 105, 113-132. 5

Atay-Kayis, A. and H. Massam (2005). A Monte Carlo method for computing the marginal likelihood in nondecomposable Gaussian graphical models. Biometrika 92, 317-335. MR2201362. doi: https://doi.org/10.1093/biomet/92.2.317. 4

Barber, R. F. and M. Drton (2015). High-dimensional Ising model selection with Bayesian information criteria. Electronic Journal of Statistics 9, 249-275. MR3326135. doi: https://doi.org/10.1214/15-EJS1012. 3

Bojinov, I. and N. Shephard (2017). Time series experiments and causal estimands: exact randomization tests and trading. Arxiv:1706.07840v2. 2,6

Bonhomme, S. and U. Sauder (2011). Recovering distributions in difference-indifferences models: A comparison of selective and comprehensive schooling. The Review of Economics and Statistics 93(2), 479-494. 5

Brodersen, K. H., F. Gaullusser, J. Koehler, N. Remy, and S. L. Scott (2015). Inferring causal impact using Bayesian structural time-series models. The Annals of Applied Statistics 9, 247-274. MR3341115. doi: https://doi.org/10.1214/14-A0AS788. 2, 3,5

de Jong, P. (1991). The diffuse Kalman filter. The Annals of Statistics 19, 1073-1083. MR1105863. doi: https://doi.org/10.1214/aos/1176348139. 4

de Jong, P. and S. Chu-Chun-Lin (1994). Stationary and non-stationary state space models. Journal of Time Series Analysis 15, 151-166. MR1263887. doi: https://doi.org/10.1111/j.1467-9892.1994.tb00182.x. 4

Ding, P. and F. Li (2017). Causal inference: A missing data perspective. Statistical Science (to appear). 5, 6

Donald, S. G. and K. Lang (2007). Inference with difference-in-differences and other panel data. The Review of Economics and Statistics 89, 221-233. 5

Doudchenko, N. and G. W. Imbens (2016). Balancing, regression, difference-indifferences and synthetic control method: A synthesis. NBER Working Paper No. 22791. 5 
Durbin, J. and S. J. Koopman (2002). A simple and efficient simulation smoother for state space time series analysis. Biometrika 89, 603-615. MR1929166. doi: https://doi.org/10.1093/biomet/89.3.603. 3, 4, 16

Durbin, J. and S. J. Koopman (2012). Time Series Analysis by State Space Methods: Second Edition. Great Clarendon Street, Oxford OX2 6DP: Oxford University Press. MR3014996. doi: https://doi.org/10.1093/acprof:oso/9780199641178.001. 0001. 3

Galindo-Garre, F. and J. K. Vermunt (2006). Avoiding boundary estimates in latent class analysis by Bayesian posterior mode estimation. Behaviormetrika 33, 43-59. MR2518758. doi: https://doi.org/10.2333/bhmk.33.43. 4

Gelfand, A. E., A. F. M. Smith, and T.-M. Lee (1992). Bayesian analysis of constrained parameter and truncated data problems using Gibbs sampling. Journal of the American Statistics Association 87, 523-532. MR1173816. 4

George, E. I. and R. E. McCulloch (1993). Variable selection via Gibbs sampling. Journal of the American Statistical Association 88 (423), 881-889. 10

Gunn, L. H. and D. B. Dunson (2005). A transformation approach for incorporating monotone or unimodal constraints. Biostatistics 6, 434-449. 4

Harvey, A. C. and S. Peters (1990). Estimation procedures for structural time series models. Journal of Forecasting 9, 89-108. 3

Holland, P. W. (1986). Statistics and causal inference. Journal of the American Statistical Association 81, 945-960. MR0867618. 5

Imbens, G. W. and D. B. Rubin (2015). Causal Inference for Statistics, Social, and Biomedical Sciences: An Introduction. New York, NY, USA: Cambridge University Press. MR3309951. doi: https://doi.org/10.1017/CB09781139025751. 5

Khare, K., B. Rajaratnam, and A. Saha (2015). Bayesian inference for Gaussian graphical models beyond decomposable graphs. Arxiv:1505.00703. 4

Koopman, S. J. (1997). Exact initial Kalman filtering and smoothing for nonstationary time series models. Journal of the American Statistical Association 92, 1630-1638. MR1615271. doi: https://doi.org/10.2307/2965434. 4

Lauritzen, S. L. (1996). Graphical Models. New York, USA: Oxford University Press Inc. MR1419991. 4

Li, F., T. Zhang, Q. Wang, M. Z. Gonzalez, E. L. Maresh, and J. A. Coan (2015). Spatial Bayesian variable selection and grouping for high-dimensional scalaron-image regression. The Annals of Applied Statistics 9, 687-713. MR3371331. doi: https://doi.org/10.1214/15-AOAS818. 3

Mitsakakis, N., H. Massam, and M. D. Escobar (2011). A Metropolis-Hastings based method for sampling from the G-Wishart distribution in Gaussian graphical models. Electronic Journal of Statistics 5, 18-30. MR2763796. doi: https://doi.org/ 10.1214/11-EJS594. 4 
Mohammadi, A. and E. C. Wit (2015). Bayesian structure learning in sparse Gaussian graphical models. Bayesian Analysis 10, 109-138. MR3420899. doi: https://doi.org/10.1214/14-BA889. 4

Ning, B., S. Ghosal, and J. Thomas (2019a). Supplement to "Bayesian method for causal inference in spatially-correlated multivariate time series". Bayesian Analysis. doi: https://doi.org/10.1214/18-BA1102SUPPA. 11, 13, 21

Ning, B., S. Ghosal, and J. Thomas (2019b). R code for "Bayesian method for causal inference in spatially-correlated multivariate time series". Bayesian Analysis. doi: https://doi.org/10.1214/18-BA1102SUPPB. 25

Ročková, V. and E. I. George (2014). EMVS: The EM approach to Bayesian variable selection. Journal of the American Statistical Association 109 (506), 828-846. MR3223753. doi: https://doi.org/10.1080/01621459.2013.869223. 4, 10, 14

Rosenbaum, P. R. (2007). Interference between units in randomized experiments. Journal of the American Statistical Association 102, 191-200. MR2345537. doi: https://doi.org/10.1198/016214506000001112. 6

Roverato, A. (2000). Cholesky decomposition of a hyper inverse Wishart matrix. Biometrika 87, 99-112. MR1766831. doi: https://doi.org/10.1093/biomet/87. 1.99. 4

Roverato, A. (2002). Hyper inverse Wishart distribution for non-decomposable graphs and its application to Bayesian inference for Gaussian graphical models. Scandinavian Journal of Statistics 29, 391-411. MR1925566. doi: https://doi.org/10.1111/ 1467-9469.00297. 4

Roy, A., T. S. McElroy, and P. Linton (2016). Constrained estimation of causal invertible VARMA models. Statistica Sinica (to appear). 4, 9, 10

Rubin, D. B. (1974). Estimating causal effects of treatments in randomized and nonrandomized studies. Journal of Educational Psychology 66, 688-701. 5, 6

Rubin, D. B. (1977). Assignment to treatment group on the basis of a covariate. Journal of Educational Statistics 2, 1-26. 5

Rubin, D. B. (2005). Causal inference using potential outcomes: design, modeling, decisions. Journal of the American Statistical Association 100, 322-331. MR2166071. doi: https://doi.org/10.1198/016214504000001880. 2,5

Smith, M. and L. Fahrmeir (2007). Spatial Bayesian variable selection with application to functional magnetic resonance imaging. Journal of the American Statistical Association 102, 417-431. MR2370843. doi: https://doi.org/10.1198/ 016214506000001031. 3

Stein, P. (1952). Some general theorems on iterants. Journal of Research of the National Bureau of Standards 48, 82-83. MR0047001. 9

Stuart, E. A. (2010). Matching methods for causal inference: A review and a look forward. Statistical Science 25, 1-21. MR2741812. doi: https://doi.org/10.1214/ 09-STS313. 5 
Ueda, N. and R. Nakano (1998). Deterministic annealing EM algorithm. Neural Networks 11, 271-282. 14

Uhler, C., A. Lenkoskiy, and D. Richardsz (2017). Exact formulas for the normalizing constants of Wishart distributions for graphical models. The Annals of Statistics 46 , 90-118. MR3766947. doi: https://doi.org/10.1214/17-A0S1543. 4

Wang, H. and S. Z. Li (2012). Efficient Gaussian graphical model determination under G-Wishart prior distributions. Electronic Journal of Statistics 6, 168-198. MR2879676. doi: https://doi.org/10.1214/12-EJS669. 4

\section{Acknowledgments}

We thank MaxPoint Interactive Inc. for providing funding and datasets for this research. We thank Dr. Alice Broadhead, Mark Lowe, Professor Fan Li, Professor Mike West and the referees for valuable input and suggestions. We thank Professor Anindya Roy for providing the R code for the stationarity constraint and many helpful discussions. Research of the second author is partially supported by NSF grant number DMS-1510238. 